\title{
ANOMALOUS LEFT ANTERIOR CEREBRAL ARTERY WITH HYPOPLASTIC RIGHT ANTERIOR CEREBRAL ARTERY
}

\author{
Mahajan A ${ }^{1}$, Goel $\mathbf{G}^{\mathbf{1}}$, Das $\mathbf{B}^{\mathbf{1}}$ \\ ${ }^{1}$ Department of Neurointervention surgery, Medanta, The Medicity. Gurgaon, Haryana; India.
}

Correspondence to A. Mahajan Medanta, The Medicity. Gurgaon, Haryana; 122001, India. +919779864015, drgauravgoel1@gmail.com

\begin{abstract}
We report an extremely rare anomalous variation of left anterior cerebral artery arising from the contralateral Paraclinoid Internal carotid artery with Hypoplastic right Anterior cerebral artery in a 56 years old female which was incidentally detected on computed tomographic angiography of cerebral vessels. Angiographic identification and characterisation of this anomaly is very important in developing a differential diagnosis and when planning a surgery and endovascular procedure.
\end{abstract}

Key words: Anterior cerebral artery, Paraclinoid Internal carotid artery, Anterior communicating artery Computed tomography angiography

\section{INTRODUCTION}

The anterior cerebral artery (ACA) usually arises from the Internal carotid artery (ICA) terminus and extends anteromedially to join the Anterior communicating artery (ACOA). There are many anomalies of A1 segment ACA described in the literature by several authors (Burbank et al., 2005; Lasjaunias et al., 2001; Murai et al., 2005; Raybaud, 2010; Raybaud, 1990; Sincoff et al., 2003). These anomalies include hypoplastic or aplastic arteries, fenestration of ACA, accessory or duplicated
ACA and persistent primitive olfactory artery (Okahara et al., 2002). However, ICA origin of contralateral ACA is extremely rare anomaly. To our best of knowledge, only three cases of anomalous origin of contralateral ACA from ICA has been reported in the literature (Burbank et al., 2005, Murai et al., 2005; Sincoff et al., 2003). Our case is unique from the three previously reported cases in having ACA origin from the contralateral paraclinoid ICA with hypoplastic A1 segment of right ACA.

\section{CASE REPORT}

A 56 years old female known to have severe pulmonary hypertension and hypothyroidism presented with sudden onset of severe headache followed by multiple episodes of vomiting for two days. She also had difficulty in maintaining balance while walking. She had a history of mitral valve replacement and was taking oral anticoagulants. Non contrast computed tomography (NCCT) was done which shows acute subdural haemorrhage along the left cerebellar hemisphere (Figure 1A). Although oral anticoagulant was anticipated as a cause of bleed in this patient, angiography and venography were done to exclude any vascular abnormality. Computed tomography angiography (CTA) and Computed tomography venography were done which confirmed no evidence of aneurysm, vascular malformation and cerebral venous sinus thrombosis. There was an incidental anomalous variation of left anterior cerebral artery arising from the right paraclinoid internal carotid artery and right ACA is hypoplastic arising from the right ICA terminus (Figure $1 \mathrm{~B}, \mathrm{C}$ and $\mathrm{D}$ ). The patient was treated conservatively for acute subdural hemorrhage.

Submitted $7^{\text {th }}$ February 2018, Accepted $9^{\text {th }}$ February 2018. Published online $28^{\text {th }}$ February 2018. To cite: Mahajan A, Goel G, Das B. 2018. Anomalous Left Anterior cerebral artery with hypoplastic right Anterior cerebral artery. Anatomy Journal of Africa. 7: $1138-1140$. 


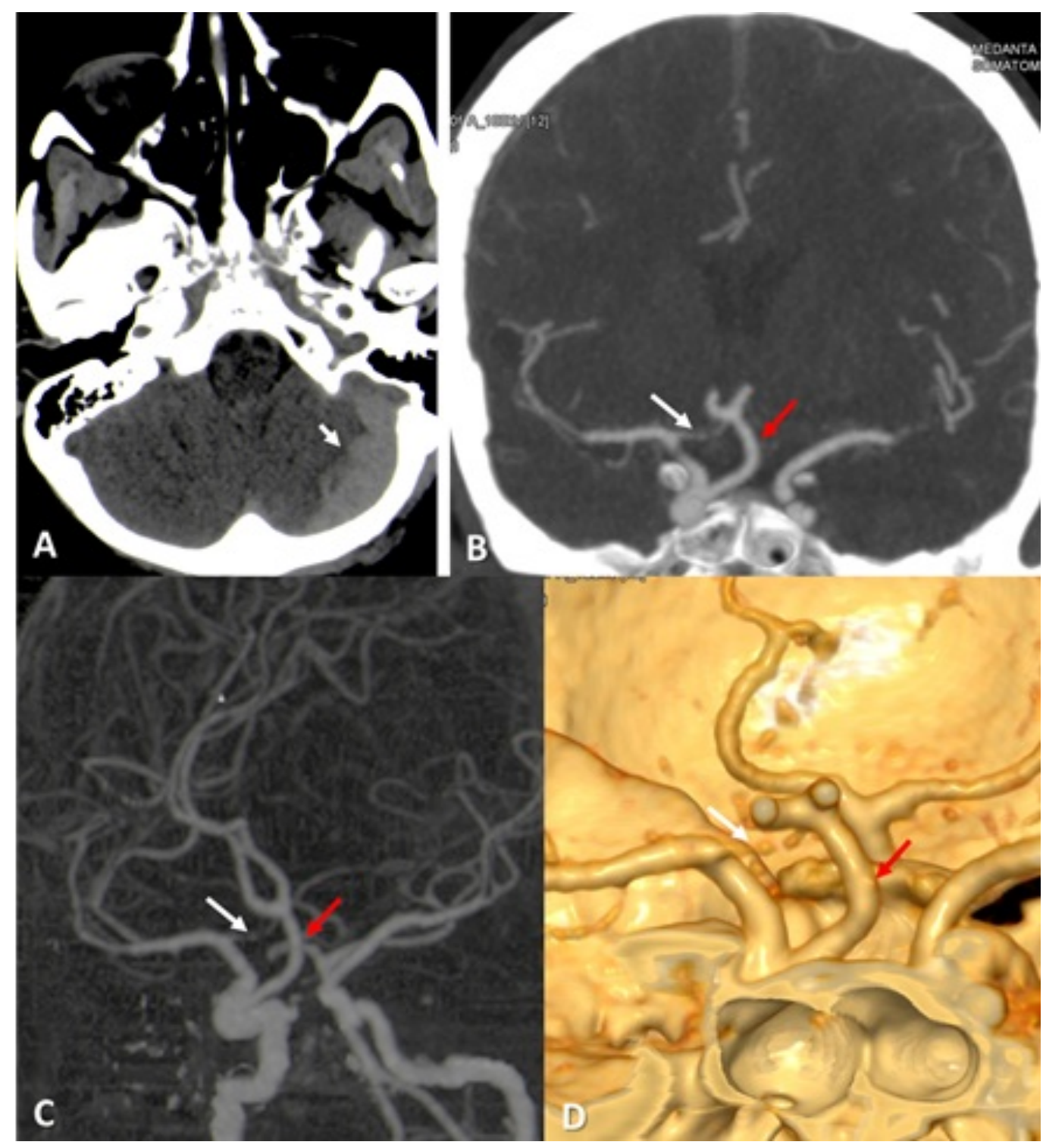

Fig 1: A Computed tomography of head shows acute subdural hemorrhage (white short arrow) along the left cerebellar hemisphere. B,C,D Coronal maximum-intensity-projection (MIP) image and 3 Dimensional Volume rendering images of CT angiography shows origin of left anterior cerebral artery from the right paraclinoid Internal carotid artery (Red arrow) and right anterior cerebral artery is hypoplastic (white arrow) arising from the right Internal carotid artery terminus.

\section{DISCUSSION}

ACA ( $\mathrm{A} 1$ segment) is one of the most frequent site for anomalous variation in circle of Willis. During the embryonic development, the cranial and caudal divisions of the ICA existed. Primitive olfactory artery formed from cranial division of the ICA and gave off two branches MCA and anterior choroidal artery. When the embryo reaches $11.5-18 \mathrm{~mm}$ stage, the primitive olfactory artery has two branches one to the nasal fossa, and the other one passing more medially to form the future ACA. Later, this artery joins with its opposite fellow artery by plexiform anastomosis which in future form anterior communicating artery. Thereafter, POA gradually shrink in size (Okahara et al., 2002). The ACA usually arises from the ICA bifurcation in most of the cases. It then extends anteromedially above the ipsilateral optic nerve or chiasm to join the anterior communicating artery (ACOA). The ACA (A1 segment) anomalies are very common with approximately $80 \%$ of the patients showing asymmetry of the A1 segment of both ACA. Other less common anomalies of A1 segment 
of ACA are fenestrated ACA, accessory or duplicated ACA and persistent primitive olfactory artery (Baptista, 1963; Burbank et al., 2005; Lasjaunias et al., 2001; Okahara et al., 2002). Although there are numerous case reports in the literature showing ACA origin from the ipsilateral ICA, ACA origin from the contralateral ICA is extremely rare anomaly (Given et al., 2002; Spinnato et al.,1999). In addition to our case report, three other cases has been reported in the literature. Murai et al., 2005 reported a case of unilateral origin of both ACA from right internal carotid artery. Burbank et al., 2005; Sincoff et al., 2003. reported anomalous contralateral origin of the left ACA (A1 segment) near the origin of the ophthalmic artery with co-dominant ACA. Our case is unique from the above-mentioned case in having ACA origin from the contralateral paraclinoid ICA with hypoplastic A1 segment of right ACA. Although, these ACA anomalies are incidentally detected in angiography their association with other coexistent anomalies including aneurysm, AVM, Moya-Moya disease and compressive symptoms of optic apparatus have been well documented in literature (Given et al., 2002; Singer et al., 1997). A thorough study of the angiograms is advisable to detect this variation and should be kept in mind before approaching the anterior communicating artery complex. In conclusion an anomalous left anterior cerebral artery with a hypoplastic contralateral vessel is extremely rare. Knowledge of these variations is crucial to the neurosurgeon and endovascular surgeon in planning and performing the Acom aneurysm surgery.

Conflict of interest: The authors declare that they have no conflict of interest.

\section{REFERENCES}

1. Baptista AG. 1963. Studies on the arteries of the brain: II-The anterior cerebral artery: Some anatomic features and their clinical implications. Neurology 13: 825-835.

2. Burbank NS, Morris PP. 2005. Unique anomalous origin of the left anterior cerebral artery. AJNR Am J Neuroradiol 26 (10):2533-5.

3. Given CA, Morris PP. 2002. Recognition and importance of an infraoptic anterior cerebral artery: case report. AJNR Am J Neuroradiol 23:452-454.

4. Lasjaunias $P$, Berenstein A, Ter Brugge KG. 2001. Surgical neuroangiography. Clinical vascular anatomy and variations. 2nd ed. 578-610. Springer-Verlag, Berlin. Pp 345 - 347.

5. Lasjaunias P, Santoyo-Vazquez A. 2001: Surgical Neuroangiography. Part 1: Clinical Vascular Anatomy and Variations (ed 2). Berlin, Springer-Verlag. 578 - 580.

6. Murai Y, Ikeda Y, Sato H, et al. 2005. Anomalous internal carotid anastomosis to contralateral anterior cerebral artery. Can J Neurol Sci 32 (3):359-60.

7. Okahara M, Kiyosue $\mathrm{H}$, Mori $\mathrm{H}$, et al. 2002. Anatomic variations of the cerebral arteries and their embryology: a pictorial review. Eur Radiol 12 (10):2548-61.

8. Raybaud C. 2010: Normal and abnormal embryology and development of the intracranial vascular system. Neurosurg Clin N Am 21:399-426.

9. Raybaud CA. 1990: Development of the arterial supply to the brain tissue, in Lasjaunias $P$, Berenstein A (eds): Surgical Neuroangiography, vol 3. New York, Springer Verlag. 1-13

10. Sincoff EH, Abdulrauf SI. 2003. Anomalous origin of the left A1 segment from the right internal carotid artery. Case illustration. J Neurosurg 99 (6):1101.

11. Singer RJ, Abe T, Taylor WH, et al. 1997. Intracavernous anterior cerebral artery origin with associated arteriovenous malformations: a developmental analysis: case report. Neurosurgery 40:829-831.

12. Spinnato S, Pasqualin A, Chioffi $F$, et al. 1999. Infraoptic course of the anterior cerebral artery associated with an anterior communicating artery aneurysm: anatomic case report and embryological considerations. Neurosurgery 44:1315-1319. 